La Corte Internacional de Justicia y el desarrollo del Derecho Internacional Ambiental

\author{
Elvis Ojeda Huerta
}

En el presente artículo, el autor analiza el surgimiento del Derecho Internacional Ambiental como una rama autónoma del Derecho Internacional en el marco de la jurisprudencia de la Corte Internacional de Justicia (CIJ). Así, profundiza respecto a los daños ambientales derivados del uso de armas nucleares y las obligaciones consuetudinarias estatales en materia ambiental con respecto a los territorios del resto de Estados. Finalmente, desarrolla reciente jurisprudencia de la $\mathrm{CIJ}$ que incorpora nuevas normas jurídicas en cuanto al daño transfronterizo.

Bachiller en Derecho por la Universidad del Pacífico. Estudios en la Academia de Derecho Internacional de La Haya y en el Instituto de Estudios Políticos de París (Sciences Po). Actualmente cursa la maestría en Diplomacia y Relaciones Internacionales de la Academia Diplomática del Perú "Javier Pérez de Cuéllar". 


\section{La Corte Internacional de Justicia y el desarrollo del Derecho Internacional Ambiental}

\section{Introducción}

El Derecho Internacional Ambiental (DIA) es una rama del Derecho Internacional relativamente nueva ${ }^{1}$. La "novedad" de esta disciplina se refleja en el número reducido de casos relacionados al DIA resueltos por la Corte Internacional de Justicia (CIJ) ${ }^{2}$. Si bien dicha cantidad ha aumentado durante los últimos años, ésta todavía no es significativa en comparación con otras áreas del Derecho Internacional ${ }^{3}$. No obstante lo anterior, el rol de la CIJ en la resolución de dichos casos ha sido relevante. La Corte ha contribuido a identificar, esclarecer o confirmar el estatus legal de algunos principios importantes del Derecho Internacional Ambiental ${ }^{4}$.

El presente artículo tiene por finalidad comprender de qué manera ha sido la CIJ aportó al desarrollo del Derecho Internacional Ambiental. Para este propósito, a lo largo del presente trabajo se analizarán los fallos y las opiniones consultivas que generaron un impacto significativo en el surgimiento de esta área. Luego, realizaremos un estudio integrado de la jurisprudencia de la CIJ relacionada al DIA que permitirá comprender su impacto en la evolución de esta disciplina.

Si bien la jurisprudencia relacionada al DIA es reciente, la hipótesis de este trabajo es que la contribución de la CIJ se fundamenta en conceptos y principios de Derecho Internacional que se consolidaron varios años atrás. En este trabajo demostraremos que los aportes de la CIJ al Derecho Internacional Ambiental son consecuencia de un largo proceso que se remonta a sus primeros fallos, toda vez que se sustentan en principios de Derecho Internacional existentes en esa época, cuando todavía el DIA no existía como una rama independiente.

$1 \quad$ El Derecho Internacional Ambiental se forjó a partir de la década de 1970 "cuando surgió como una posible subdisciplina del derecho internacional público". Oliveira Do Prado, Rafael, La ecologización de la Corte Internacional de Justicia, Anuario Mexicano de Derecho Internacional, vol. XI, 2011, pp. 45-76, p. 70.

2 Fitzmaurice, Malgosia, The International Court of Justice and International Environmental Law. En: The Development of International Law by the International Court of Justice, editado por TAMS, Christian y SLOAN, James, Oxford University Press, 2013, pp. 353374, p. 353.

3 A manera de ejemplo podemos mencionar el gran desarrollo jurisprudencial de la CIJ en áreas tales como la responsabilidad internacional o la protección diplomática.

4 OWADA, Hisashi. International Environmental Law and the International Court of Justice. Inaugural Lecture at the Fellowship Programme on International and Comparative Environmental Law, Iustum Aequum Salutare, II. 2006/3-4, p. 5-32, p. 10. 


\section{Los cimientos - canal de Corfú y Barcelona traction}

La contribución de la Corte Internacional de Justicia se inició con decisiones que resolvieron casos no relacionados con el Derecho Internacional Ambiental ${ }^{5}$. El aporte realizado durante esta etapa estuvo concentrado principalmente en analizar las consecuencias de los daños transfronterizos ${ }^{6}$ y resaltar los efectos erga omnes de ciertas obligaciones internacionales. El concepto del daño transfronterizo fue confirmado por la CIJ en el asunto relativo al Canal de Corfút. El fallo de fecha 9 de abril de 1949 estableció que los Estados tienen la obligación "de no permitir a sabiendas que su territorio sea usado para realizar actos contrarios a los derechos de otros Estados" ${ }^{\prime \prime}$.

Esta afirmación es relevante no solo porque confirmó un principio de Derecho Internacional -conocido como "sic utere tuo ut alienum non laedas" ${ }^{\prime 9}$ - que había sido utilizado por otros tribunales internacionales con anterioridad (por ejemplo, en el caso Trail Smelter) ${ }^{10}$, sino también porque la CIJ estableció que dicho principio formaba parte del Derecho Internacional general ${ }^{11}$. Al respecto, es importante mencionar que el ámbito de aplicación de este principio es muy amplio, puesto que el mismo puede ser

$5 \quad$ Fitzmaurice, Malgosia, The International Court of Justice and International Environmental Law, En: The Development of International Law by the International Court of Justice, editado por Tams, Christian y Sloan, James, Oxford University Press, 2013. p. 355.

6 Aquellos daños que se producen más allá de las fronteras del Estado donde el daño se origina. En: Pastor Ridruejo, José Antonio, Curso de Derecho Internacional Público y organizaciones Internacionales, Tecnos, 19 edición, 2015, p. 195; El origen del daño se localiza en el territorio y otros lugares bajo la jurisdicción o el control del Estado y los efectos perjudiciales se producen sobre personas, bienes o el medio ambiente en otro Estado. En: Cassase, Antonio, International Law, Segunda edición, Oxford University Press, 2005, p. 498.

7 Corfu Channel case, Judgment of April 9th, 1949: I.C.J. Reports 1949, p.4.

8 Corfu Channel case, Judgment of April 9th, 1949: I.C.J. Reports 194, p. 22.

9 El principio también es conocido como "principe de l'utilisation non dommageable du territoire". Quoc Dinh, Nguyen, Droit International Public, Séptima edición, París: LGDJ, 2002, p. 1275.

10 En el caso Trail Smelter el tribunal arbitral señaló que "Under the principles of international law, as well as the law of the United States no State has the right to use or permit the use of its territory in such a manner as to cause injury by fumes in or to the territory of another or the properties or persos therein, when the case is of serious consequence and the injury is established by clear and convincing evidence". Recueil des Sentences Arbitrales. vol. III, Naciones Unidas, pp. 1906-1982.

11 La CIJ mencionó que las obligaciones de Albania surgieron de ciertos "principios generales y bien reconocidos" por el Derecho Internacional general. Corfu Channel case, Judgment of April 9th, 1949: I.C.J. Reports 194, p.22. 
utilizado en distintas áreas del Derecho Internacional. Igualmente, durante la época en que se dio este fallo todavía no estaba claro si la protección ambiental era requerida como tal o solo cuando se producía un perjuicio a un Estado como consecuencia de conductas realizadas en otro Estado ${ }^{12}$. No existía certeza de la necesidad de un componente estatal en la configuración de la obligación de no dañar el medio ambiente.

Del análisis de los fallos anteriores al Canal de Corfú inferimos que para el Derecho Internacional de esa época el medio ambiente no tenía un valor en sí mismo. Si bien Cassese resalta que uno de los tribunales arbitrales hizo referencia a los recursos naturales y aludió a intereses comunes, ${ }^{13}$ la idea de que el medio ambiente tenía un valor intrínseco que debía ser protegido, incluso cuando no existiera un perjuicio en contra de algún Estado, aún no se había desarrollado ${ }^{14}$. Podemos afirmar entonces que la contraparte de la primera contribución es que no se considera la protección del medio ambiente por su valor intrínseco, pues existía una concepción distinta a la idea del medio ambiente como un bien común internacional a ser preservado por todos los Estados, concepto que fue desarrollado con posterioridad ${ }^{15}$.

Si bien el fallo del Canal de Corfú consagró una visión limitada del principio sic utere tuo ut alienum non laedas, ya que solo contempló aquellas obligaciones ambientales entre Estados, no se debe subestimar el impacto de esta decisión. El principio reconocido en la decisión de la CIJ tuvo un papel formativo en el surgimiento del DIA como un área específica del Derecho Internacional y sirvió como base para la celebración de ciertos instrumentos fundadores de esta disciplina. ${ }^{16}$

El segundo concepto formulado durante esta etapa lo encontramos en el caso Barcelona Traction ${ }^{17}$. El fallo de la CIJ del 5 de febrero de 1970 incluyó un obíter

12 Cassese, Antonio, International Law, Oxford University Press, 2001, p. 377.

13 Cassese, Antonio, International Law, Oxford University Press, 2001, p. 377.

14 Viñuales, Jorge, The Contribution of the International Court of Justice to the Development of International Environmental Law: A Contemporary Assessment, Fordham International Law Journal, Volume 32, Issue 1, Article 14, 2008, pp. 232 - 258, p. 238.

Sohnle, Jochen, Irruption du droit de l'environnement dans la jurisprudence de la C.I.J.; l'affaire Gabvikovo-Nagymaros, 102 Revue Générale de Droit International Publique, 1998 , p. 85.

Por ejemplo, la Declaración de la Conferencia de Naciones Unidas sobre el Medio Humano, la Declaración de Rio sobre Medio Ambiente y Desarrollo, el Convenio sobre la contaminación atmosférica transfronteriza a gran distancia, el Convenio de Viena para la Protección de la Capa de Ozono, la Convención Marco de Naciones Unidas sobre Cambio Climático (CMNUCC), entre otros. p. 3. 
dictum que reconoció la existencia de obligaciones internacionales con efectos erga omnes. La CIJ aseguró que:

"(...) debe establecerse, en particular, una distinción esencial entre las obligaciones de los Estados hacia la comunidad internacional en su conjunto y las que nacen respecto de otro Estado en el campo de la protección diplomática. Por su propia naturaleza, las primeras conciernen a todos los Estados. Dada la importancia de los derechos en cuestión, puede considerarse que todos los Estados tienen un interés jurídico en que tales derechos sean protegidos; las obligaciones de que se trata son obligaciones erga omnes"18.

La Corte Internacional de Justicia, al reconocer por primera vez el concepto de las obligaciones erga omnes, ${ }_{1}^{19}$ tomó un paso importante en la evolución del DIA. Dicho concepto sirvió de base para el desarrollo posterior de normas relacionadas a la protección del ambiente por su valor intrínseco, indistintamente de la existencia de un perjuicio a un Estado en concreto $^{20}$. Conforme la comunidad internacional adquiere mayor conciencia sobre los riesgos medioambientales, el principio de erga omnes tomará más relevancia ${ }^{21}$. Con el paso de los años esta concepción permitirá pasar de una construcción de obligaciones medioambientales a nivel interestatal a una que acoge nociones de intereses colectivos ${ }^{22}$.

Podemos mencionar entonces que las principales contribuciones de los casos antes analizados son el reconocimiento del principio que obliga al Estado a no permitir que su territorio sea utilizado para generar un perjuicio en otros Estados y la identificación de las obligaciones internacionales erga omnes. Tal como veremos más adelante, estas contribuciones sirvieron como fundamento para el desarrollo posterior del DIA, ya que Justice, editado por Tams, Christian y Sloan, James, Oxford University Press, 2013.

Barcelona Traction, Light and Power Company, Limited, Judgment, I.C.J. Reports 1970, pár. 33.

Owada, Hisashi, International Environmental Law and the International Court of Justice. Inaugural Lecture at the Fellowship Programme on International and Comparative Environmental Law, Iustum Aequum Salutare, II. 2006/3-4, pp. 5-32, p. 12.

Viñuales, Jorge, The Contribution of the International Court of Justice to the Development of International Environmental Law: A Contemporary Assessment, Fordham International Law Journal, Volume 32, Issue 1, Article 14, 2008, p. 239.

Fitzmaurice, Malgosia. The International Court of Justice and International Environmental Law. p. 358, En: The Development of International Law by the International Court of Justice, editado por Tams, Christian y Sloan, James, Oxford University Press, 2013.

Fitzmaurice, Malgosia, The International Court of Justice and International Environmental 
fueron aplicados a los casos directamente relacionados con cuestiones ambientales ${ }^{23}$. Ambos principios - también llamados "pilares de la protección ambiental"24 - sentaron las bases de la responsabilidad internacional por daños ambientales transfronterizos y de la protección del medio ambiente como tal ${ }^{25}$.

\section{Primeros casos ambientales - ensayos nucleares I}

Los primeros casos relacionados con preocupaciones ambientales son los relativos a los Ensayos Nucleares ${ }^{26}$ durante la década de 1970. Australia y Nueva Zelanda iniciaron procedimientos contra Francia por considerar que las pruebas nucleares en la atmósfera realizadas en la Polinesia Francesa emitían altos índices de radioactividad que generaban efectos nocivos en sus poblaciones y medio ambiente ${ }^{27}$. Ambos demandantes solicitaron a la CIJ que ordene a Francia la conclusión de dichos ensayos y que declare que estos eran incompatibles con las normas aplicables al derecho internacional ${ }^{28}$.

Resulta relevante el contexto en el cual se llevaron a cabo estos procesos. En 1972 se celebró la Conferencia de Naciones Unidas sobre el Medio Humano en Estocolmo. Esta reunión -llamada Cumbre de la Tierra - constituyó la primera gran conferencia en el marco de las Naciones Unidas que se ocupó sobre cuestiones de carácter ambiental ${ }^{29}$. En opinión de Baylis y Smith, la Cumbre de la Tierra significó un "punto de inflexión"30 en el desarrollo de la política internacional del medio ambiente. De otro lado, es

23 Viñuales, Jorge, The Contribution of the International Court of Justice to the Development of International Environmental Law: A Contemporary Assessment, Fordham International Law Journal, Volume 32, Issue 1, Article 14, 2008, p. 235.

Viñuales, Jorge, The Contribution of the International Court of Justice to the Development of International Environmental Law: A Contemporary Assessment, Fordham International Law Journal, Volume 32, Issue 1, Article 14, 2008, p. 236.

Horbach, N. y Bekker, P. State Responsibility for Injurious Transboundary Activity in Retrospect. Netherlands International Law Review, vol. 50, Holanda, 2003, p. 333. Tests (Australia v. France), Judgment, I.C.J. Reports 1974, p. 253.

Nuclear Tests (New Zealand v. France), Judgment, I.C.J. Reports 1974, p. 457; Nuclear Tests (Australia v. France), Judgment, I.C.J. Reports 1974, pár. 1.

Nuclear Tests (New Zealand v. France), Judgment, I.C.J. Reports 1974, p. 457; Nuclear Tests (Australia v. France), Judgment, I.C.J. Reports 1974, pár. 11.

Stakeholder, Forum, Environmental Institutions for the 21st Century: An International Court for the Environment, ICE Coalition, 2011, p. 5.

Baylis, John y Smith, Steve, The Globalization of World Politics, Tercera edición, 2005, Oxford University Press, p. 454. 
relevante resaltar que ambos procesos se desarrollaron en el marco de un movimiento general en contra de los ensayos nucleares atmosféricos ${ }^{31}$.

A pesar de que los argumentos estuvieron sustentados principalmente en la vulneración de la soberanía en aplicación del principio "sic utere tuo ut alineum non laedas", ${ }^{32}$ los demandantes argumentaron que Francia había incurrido en responsabilidad internacional por vulnerar una norma emergente de derecho consuetudinario que prohibía la contaminación al medio ambiente. Los reclamos se basaban en parte en la contaminación que los ensayos nucleares podían causar al ambiente en la región ${ }^{33}$. Dicho argumento se basó en el principio 21 de la Declaración de Estocolmo de 1972 que establece que:

"De conformidad con la carta de las Naciones Unidas y con los principios del derecho internacional, los Estados tienen el derecho soberano de explotar sus propios recursos en aplicación de su propia política ambiental, y la obligación de asegurarse de que las actividades que se lleven a cabo dentro de su jurisdicción o bajo su control no perjudiquen al medio ambiente de otros Estados o de zonas situadas fuera de toda jurisdicción nacional ${ }^{134}$.

El principio reconocido en el caso del Canal de Corfú sirvió de base para la elaboración del principio 21 de la Declaración de Estocolmo. No obstante, a diferencia de lo expresado por la $\mathrm{CIJ}$, el texto de la Declaración está redactado de tal manera que la protección ambiental incluye aquellas zonas situadas fuera de toda jurisdicción nacional. En este sentido, podemos afirmar que la Declaración de Estocolmo va más allá de lo expresado por la CIJ puesto que en Canal de Corfú no se hizo ninguna referencia a la protección del medio ambiente como tal.

La interrogante respecto al carácter consuetudinario de dicha norma se mantuvo abierta ya que la CIJ no llegó a analizar el fondo del asunto ${ }^{35}$. La Corte desperdició la

31 Resolución de la Asamblea General de la ONU 2934 (XXVII). Urgente necesidad de suspender los ensayos nucleares y termonucleares. 29 de noviembre de 1972.

32 Owada, Hisashi. International Environmental Law and the International Court of Justice. Inaugural Lecture at the Fellowship Programme on International and Comparative Environmental Law, Iustum Aequum Salutare, II. 2006/3-4, pp. 5-32, p. 16.

33 Owada, Hisashi. International Environmental Law and the International Court of Justice. Inaugural Lecture at the Fellowship Programme on International and Comparative Environmental Law, Iustum Aequum Salutare, II. 2006/3-4, pár. 26.

A partir de las declaraciones unilaterales del presidente y el ministro de Defensa de Francia, que hicieron público la intención de cesar los ensayos nucleares en la atmósfera, la CIJ estimó que las demandas se habían quedado sin objeto, lo que no daba lugar a ninguna decisión de la Corte. 
oportunidad de esclarecer si existía alguna norma jurídica que prohibiera la contaminación del medio ambiente por su valor intrínseco o, en todo caso, si la obligación se limitaba solo a aquellas situaciones en las que se perjudicaba a un Estado en específico ${ }^{36}$. Hasta la fecha del fallo de Ensayos Nucleares ningún tribunal internacional había desarrollado esta concepción ${ }^{37}$. Sin embargo, a partir de las opiniones individuales de los jueces de la Corte, que eran contrarias entre sí, ${ }^{38}$ podemos inferir que si una norma consuetudinaria existía en ese momento, esta estaría limitada solo a la contaminación transfronteriza ${ }^{39}$.

\section{Tomando conciencia - armas nucleares, ensayos nucleares II $\mathrm{V}$ gabcikovo-nagymaros}

Con el transcurso de los años la preocupación de los Estados por los problemas medioambientales se incrementó ${ }^{40}$. En 1994 la Asamblea General de las Naciones Unidas solicitó a la CIJ que dé con carácter de urgencia una opinión consultiva sobre la siguiente pregunta: "¿Autoriza el derecho internacional en alguna circunstancia la amenaza o el empleo de armas nucleares?"41.

En la Opinión Consultiva Sobre la licitud de la amenaza o del empleo de armas nucleares $^{42}$ del 8 de julio de 1996, la CIJ considero que si bien el derecho internacional relativo a la protección del medio ambiente no proscribe el empleo de las armas

Oliveira Do Prado, Rafael, La ecologización de la Corte Internacional de Justicia, Anuario Mexicano de Derecho Internacional, vol. XI, 2011, pp. 45-76, p. 54.

Viñuales, Jorge. The Contribution of the International Court of Justice to the Development of International Environmental Law: A Contemporary Assessment, Fordham International Law Journal, Volume 32, Issue 1, Article 14, 2008, p. 240

Opinión disidente del juez de Castro, párrafos 4 y 5; Opinión individual del del juez Petrén p. 306.

Viñuales, Jorge. The Contribution of the International Court of Justice to the Development of International Environmental Law: A Contemporary Assessment, Fordham International Law Journal, Volume 32, Issue 1, Article 14, 2008, p. 240.

La celebración de la Conferencia de las Naciones Unidas sobre el Medio Ambiente y el Desarrollo, celebrada en 1992 es reflejo de esta preocupación.

Resolución de la Asamblea General de la ONU 49/75K (1994). Petición a la Corte Internacional de Justicia de una Opinión Consultiva sobre la legalidad de la amenaza o el empleo de armas nucleares. A/RES/49/75 K

Legality of the Threat of Use of Nuclear Weapons, Advisory Opinion, I.C.J. Reports 1996, p. 226. 
nucleares, estas pueden constituir una catástrofe para el medio ambiente ${ }^{43}$. La CIJ agregó además que:

"la existencia de la obligación general de que los Estados velen por que las actividades realizadas dentro de su jurisdicción o bajo su control no dañen el medio ambiente de otros Estados o zonas que estén fuera de su jurisdicción nacional forma parte ya del corpus de normas internacionales en materia de medio ambiente ${ }^{\prime \prime 44}$.

La particularidad de esta afirmación radica en que, a diferencia de la jurisprudencia anterior, la Corte tomó en consideración los componentes estatales y no estatales. En relación al componente estatal, reafirmó el principio "sic utere tuo ut alineum non laedas"desarrollado en el caso del Canal de Corfú. Respecto al componente no estatal, la CIJ reconoció por primera vez que la obligación de proteger el medio ambiente por su propio valor intrínseco constituye una obligación internacional. No es extraño entonces que esta decisión sea considerada la mayor contribución de la CIJ al Derecho Internacional Ambiental ${ }^{45}$.

El reconocimiento de ambos principios es confirmado con posterioridad en la Orden del 22 de septiembre de 1995 que resolvió la acción presentada por Nueva Zelanda ${ }^{46}$. En dicha acción, Nueva Zelanda argumentó que los ensayos nucleares subterráneos realizados por Francia en los atolones Mururoa y Fangataufa configuraban hechos internacionalmente ilícitos, ${ }^{47}$ puesto que ocasionaron un impacto negativo en el medio ambiente.

Si bien la Orden desestimó la aplicación de Nueva Zelanda, la CIJ formuló su conclusión "sin perjuicio de las obligaciones de los Estados de respetar y proteger el

43 Legality of the Threat of Use of Nuclear Weapons, Advisory Opinion, I.C.J. Reports 1996, pár. 29.

Legality of the Threat of Use of Nuclear Weapons, Advisory Opinion, I.C.J. Reports 1996, pár. 29.

Viñuales, Jorge. The Contribution of the International Court of Justice to the Development of International Environmental Law: A Contemporary Assessment, Fordham International Law Journal, Volume 32, Issue 1, Article 14, 2008, p. 234. Court's Judgment of 20 December 1974 in the Nuclear Tests (New Zealand v. France) Case, I.C.J. Reports 1995, p. 288.

Request for an Examination of the Situation in Accordance with Paragraph 63 of the Court's Judgment of 20 December 1974 in the Nuclear Tests (New Zealand v. France) Case, I.C.J. Reports 1995, pár. 4. 
medio ambiente natural ${ }^{\prime \prime 4}$. Aunque esta frase haya estado dirigida específicamente a los Estados que formaban parte del proceso, consideramos que la afirmación de la CIJ tiene un carácter de alcance general, toda vez que dichas obligaciones, que no fueron determinadas, pertenecen a todos los Estados, sin importar que hayan celebrado o no un tratado particular sobre la materia ${ }^{49}$.

Esta afirmación se sustenta además en la solicitud de Nueva Zelanda, que no solo se basó en obligaciones derivadas de un tratado, sino también en aquellas que se originan en la costumbre internacional ${ }^{50}$. A diferencia de Ensayos Nucleares $I$, en el que los demandantes se centraron en reclamos basados en la vulneración de la soberanía, este caso confirma un cambio de percepción, ${ }^{51}$ debido a que Nueva Zelanda también se concentró en los preocupaciones medioambientales de la región ${ }^{52}$.

La naturaleza consuetudinaria de las obligaciones ambientales es confirmada por la CIJ en Gabcikovo-Nagymaros ${ }^{53}$. En la decisión del 25 de setiembre de $1997^{54}$ la CIJ reconoció la existencia de nuevas normas del Derecho Internacional Ambiental relevantes para la implementación de tratados, ${ }^{55}$ que pertenecen claramente al derecho internacional consuetudinario ${ }^{56}$. La afirmación se encuentra respaldada por la

48 Request for an Examination of the Situation in Accordance with Paragraph 63 of the Court's Judgment of 20 December 1974 in the Nuclear Tests (New Zealand v. France) Case, I.C.J. Reports 1995, pár. 64.

Viñuales, Jorge. The Contribution of the International Court of Justice to the Development of International Environmental Law: A Contemporary Assessment, Fordham International Law Journal, Volume 32, Issue 1, Article 14, 2008, p. 247.

Request for an Examination of the Situation in Accordance with Paragraph 63 of the Court's Judgment of 20 December 1974, op. cit., pár. 5.

51 Fitzmaurice, Malgosia. The International Court of Justice nad International Environmental Law. En : The Development of International Law by the International Court of Justice, editado por Tams, Christian y Sloan, James, Oxford University Press, 2013. p. 362

Stephens, Tim. International Courts and Environmental Protection. Cambridge Studies in International and Comparative Law, 2009, pp. 137 - 149.

Lammers, Johan. The Gabcikovo-Nagymaros Case Seen in Particular from the Perspective of the Law of International Watercourses and the Protection of the Environment. Leiden Journal of International law, volumen 11, issue 2, 1998, pp 287-320, p. 287.

Gabcikovo-Nagymaros Project (Hungary/Slovakia), Judgment, I.C.J. Reports 1997, p. 7.

Gabcikovo-Nagymaros Project, (Hungary/Slovakia), Judgment, I.C.J. Reports 1997, pár. 112.

Stec, Stec y Eckstein, Gabriel, Of Solemn Oaths and Obligations: The Environmental Impact of the ICJ's Decision in the Case Concerning the Gabcikovo-Nagymaros Project, Yearbook of International 41, volumen 8, 1997, pp. 41-50, p. 42. 
propia referencia expresa hacia el párrafo 29 de la opinión consultiva Sobre la licitud de la amenaza o del empleo de armas nucleares ${ }^{57}$.

Los últimos tres casos analizados contemplan componentes estatales y no estatales de la protección ambiental que ya disfrutaban de una base consuetudinaria. ${ }^{58} \mathrm{La}$ importancia de dichos casos radica en que la CIJ consolidó los conceptos desarrollados en la jurisprudencia previa y los adoptó en el área específica del DIA. ${ }^{59}$ La reiteración de los principios reconocidos en Armas Nucleares reconocen expresamente las crecientes preocupaciones ambientales en el derecho internacional. ${ }^{60}$ Tal como menciona Viñuales, no debemos subestimar los aportes de la CIJ al Derecho Internacional Ambiental durante la década de los noventa, ${ }^{61}$ pues se demostró que el DIA pasó a ser una parte sustancial y relevante del derecho internacional general en el transcurso de algunas décadas ${ }^{62}$.

V. Siglo XXI - plantas de celulosas, actividades en la zona fronteriza, construcción de carretera a lo largo del río san juan y fumigación aérea de herbicidas ${ }^{63}$

57 Gabcikovo-Nagymaros Project, (Hungary/Slovakia), Judgment, I.C.J. Reports 1997, pár. 53.

58 Viñuales, Jorge. The Contribution of the International Court of Justice to the Development of International Environmental Law: A Contemporary Assessment, Fordham International Law Journal, Volume 32, Issue 1, Article 14, 2008, p. 249.

Kiss, Alexandre-Charles. The International Court of Justice and the Protection of the Environment. Hague Yearbook of International Law, vol. 11, 1998, pp. 1-13.

Stephens, Tim. International Courts and Environmental Protection. Cambridge Studies in International and Comparative Law, 2009, p. 140.

Viñuales, Jorge. The Contribution of the International Court of Justice to the Development of International Environmental Law: A Contemporary Assessment, Fordham International Law Journal, Volume 32, Issue 1, Article 14, 2008, p. 233.

Fitzmaurice, Malgosia. The International Court of Justice and International Environmental Law. En: The Development of International Law by the International Court of Justice, editado por TAMS, Christian y SLOAN, James, Oxford University Press, 2013, p. 370.

En relación al caso de la Fumigación aérea de herbicidas (Ecuador c. Colombia), Ecuador inició un procedimiento contra Colombia con respecto a una controversia relativa a "la fumigación aérea de herbicidas tóxicos llevada a cabo por Colombia en lugares próximos a su frontera con el Ecuador, en dicha frontera y a través de ella" que "ya ha causado graves daños a las personas, a las cosechas, a los animales y al medio ambiente natural del lado ecuatoriano de la frontera, y plantea un serio peligro de nuevos daños a lo largo del tiempo". El 9 de septiembre de 2013 ambas partes llegaron a un Acuerdo "que resuelve de manera plena y definitiva todas las pretensiones del Ecuador contra Colombia". El agente de Ecuador comunicó a la CIJ que su Gobierno deseaba desistir del procedimiento en la causa. El 13 de septiembre de 2013 el Presidente de la CIJ dictó una 
Con el inicio del nuevo milenio la CIJ tuvo la oportunidad de aclarar y desarrollar ciertos conceptos específicos del DIA ${ }^{64}$. En el asunto relativo a las Plantas de Celulosa ${ }^{65}$ Argentina presentó una demanda contra Uruguay respecto a una controversia relativa a la supuesta violación de los términos del Estatuto del Río Uruguay de $1975^{66}$, por otorgar autorizaciones para la construcción y eventual operación de dos plantas de pasta de celulosa en la ribera izquierda del río Uruguay, que constituye el límite entre ambos Estados ${ }^{67}$. Argentina argumentó que dichas papeleras supondrían un perjuicio a la calidad de las aguas del río y a la diversidad biológica de la región ${ }^{68}$.

En el fallo del 20 de abril de 2010, la CIJ reafirmó y consolidó las líneas generales de la jurisprudencia anterior respecto al principio "sic utere tuo ut alineum non laedas ${ }^{69}$. Sobre la base del principio reconocido en Canal de Corfút ${ }^{70}$ la CIJ señaló que el principio de prevención ${ }^{71}$ es una regla del derecho internacional consuetudinario que tiene sus orígenes en la debida diligencia que se requiere de un Estado en su territorio ${ }^{72}$.

providencia por la cual se hizo constar el desistimiento del procedimiento y se dispuso que se eliminara la causa de la lista de la CIJ. Aerial Herbicide Spraying (Ecuador v. Colombia), Order of 13 September 2013, I.C.J. Reports 2013, p. 278.

Viñuales, Jorge. The Contribution of the International Court of Justice to the Development of International Environmental Law: A Contemporary Assessment, Fordham International Law Journal, Volume 32, Issue 1, Article 14, 2008, p. 234.

Pulp Mills on the River Uruguay (Argentina v. Uruguay), Judgment, I.C.J. Reports 2010, p. 14.

El Estatuto de 1975 requiere que los Uruguay y Argentina coordinen y cooperen en la administración del río Uruguay. Asimismo, establece principios sustanciales que gobiernan el uso del río. Fue celebrado el 26 de febrero de 1975.

Pulp Mills on the River Uruguay (Argentina v. Uruguay), Judgment, I.C.J. Reports 2010, pár. 1.

Casanovas, Oriol y Rodrigo, Ángel, Casos y Textos de Derecho Internacional Público. 6ta edición, editorial Tecnos, 2010, p. 739; CIJ, pár. 2.

Pulp Mills on the River Uruguay (Argentina v. Uruguay), Judgment, I.C.J. Reports 2010, pár. 101.

"La obligación de todo Estado de no permitir a sabiendas que su territorio sea usado para actos contrarios a los derechos de otros Estados". Corfu Channel case, Judgment of April 9th, 1949: I.C.J. Reports 1949, p. 22

El artículo 7 del tratado del 26 de febrero de 1975 estableció un "estatuto para el uso del río"cubriendo entre otros aspectos, la conservación de los recursos vivos y la prevención de la contaminación del río. Pulp Mills on the River Uruguay (Argentina v. Uruguay), Judgment, I.C.J. Reports 2010, pár. 75.

Pulp Mills on the River Uruguay (Argentina v. Uruguay), Judgment, I.C.J. Reports 2010, pár. 101. 
Asimismo, a partir de las disposiciones establecidas en Armas Nucleares reiteró que el Derecho Internacional consuetudinario obliga a los Estados a "usar todos los medios a su disposición para evitar que las actividades que tienen lugar en su territorio, o en cualquier área bajo su jurisdicción, causen un daño significativo al medio ambiente de otro Estado"173.

Respecto al concepto de desarrollo sostenible, la CIJ consideró que existe una interconexión entre el aprovechamiento equitativo y razonable del agua, como recurso compartido, y el equilibrio entre el desarrollo económico y la protección ambiental ${ }^{74}$. Asimismo, distinguió entre las obligaciones de naturaleza procesal y aquellas de naturaleza sustantiva. El primer grupo incluye las obligaciones de información, notificación, entre otras. En el segundo grupo se encuentran las obligaciones de contribuir al aprovechamiento óptimo y racional del rí $^{75}$, de asegurar que el manejo del suelo y de los bosques no perjudique el régimen del río o la calidad de sus aguas, ${ }^{76}$ de coordinar medidas para evitar cambios en el equilibrio ecológico, ${ }^{77}$ de prevenir la contaminación ${ }^{78}$ y preservar el medio acuático ${ }^{79}$.

Uno de los principales aportes de la sentencia es el reconocimiento de la obligación de elaborar un Estudio de Impacto Ambiental (EIA) antes de la puesta en funcionamiento de un proyecto industrial susceptible de "tener un impacto adverso significativo en un contexto extraterritorial" ${ }^{\prime 80}$. Esta obligación, que es parte del Derecho Internacional consuetudinario $^{81}$, está acompañada del monitoreo continuo de los efectos que el

73 Pulp Mills on the River Uruguay (Argentina v. Uruguay), Judgment, I.C.J. Reports 2010, pár. 101.

Siendo esta conexión "la esencia del desarrollo sustentable". Pulp Mills on the River Uruguay, op. cit., pár. 107.

Pulp Mills on the River Uruguay (Argentina v. Uruguay), Judgment, I.C.J. Reports 2010, párs. 170 - 177.

Pulp Mills on the River Uruguay (Argentina v. Uruguay), Judgment, I.C.J. Reports 2010," párs. $178-180$.

Pulp Mills on the River Uruguay (Argentina v. Uruguay), Judgment, I.C.J. Reports 2010," párs. $181-189$.

Pulp Mills on the River Uruguay (Argentina v. Uruguay), Judgment, I.C.J. Reports 2010," párs. $190-202$.

Pulp Mills on the River Uruguay (Argentina v. Uruguay), Judgment, I.C.J. Reports 2010, párs. $190-202$.

Pulp Mills on the River Uruguay (Argentina v. Uruguay), Judgment, I.C.J. Reports 2010, pár. 204.

Pulp Mills on the River Uruguay (Argentina v. Uruguay), Judgment, I.C.J. Reports 2010, pár. 205. 
proyecto produce en el medio ambiente luego de iniciadas las actividades ${ }^{82}$. Pese a que el fallo no determinó el alcance y contenido de los EIA ${ }^{83}$, consideramos que la importancia de este instrumento radica en que permite "operativizar" ${ }^{\prime 84}$ el principio que prohíbe el uso del propio territorio de tal forma que afecte el de los demás.

Los aportes de Plantas de Celulosa fueron confirmados por la CIJ en los casos Ciertas actividades Ilevadas a cabo por Nicaragua en la zona fronteriza y Construcción de una carretera en Costa Rica a lo largo del río San Juan ${ }^{85}$ el 16 de diciembre de 2015. La Corte mencionó que el principio de prevención aplica de manera general a todas aquellas actividades que puedan ocasionar daños transfronterizos ${ }^{86}$. En este sentido, la CIJ agregó que "para cumplir su obligación de ejercer la debida diligencia en la prevención de un daño ambiental transfronterizo significativo, un Estado debe (...) averiguar si existe un riesgo de un daño transfronterizo significativo ${ }^{\prime 87}$ antes de iniciar una proyecto "que tenga el potencial de afectar adversamente el medio ambiente de otro Estado" ${ }^{\prime \prime 8}$. En este sentido, la CIJ reconoció que la obligación de llevar a cabo un estudio de impacto ambiental requiere de una evaluación ex ante a la implementación de un proyecto ${ }^{89}$.

Pulp Mills on the River Uruguay (Argentina v. Uruguay), Judgment, I.C.J. Reports 2010, pár. 205.

Pulp Mills on the River Uruguay (Argentina v. Uruguay), Judgment, I.C.J. Reports 2010, pár. 216.

Saco, Víctor. El caso de las plantas de celulosa sobre el río Uruguay. Sentencia de la Corte Internacional de Justicia de 20 de abril de 2010 (Argentina contra Uruguay). Agenda Internacional, Año XVII, Nº 28, 2010, pp. 281-304, p. 303.

Ambos casos fueron acumulados mediante la Orden del 17 de abril de 2013, en aplicación del artículo 47 del Reglamento de la CIJ.

Certain Activities carried out by Nicaragua in the border area (Costa Rica v. Nicaragua) \& Construction of a Road in Costa Rica along the San Juan River (Nicaragua v. Costa Rica), I.C.J., Judgment 16 december 2015, pár. 104.

Certain Activities carried out by Nicaragua in the border area (Costa Rica v. Nicaragua) \& Construction of a Road in Costa Rica along the San Juan River (Nicaragua v. Costa Rica), I.C.J., Judgment 16 december 2015, pár. 104.

Certain Activities carried out by Nicaragua in the border area (Costa Rica v. Nicaragua) \& Construction of a Road in Costa Rica along the San Juan River (Nicaragua v. Costa Rica), I.C.J., Judgment 16 december 2015, pár. 104.

Certain Activities carried out by Nicaragua in the border area (Costa Rica v. Nicaragua) \& Construction of a Road in Costa Rica along the San Juan River (Nicaragua v. Costa Rica), I.C.J., Judgment 16 december 2015, pár. 161. 
Al igual que en Plantas de Celulosa la CIJ reiteró que le corresponde a cada Estado determinar el contenido del estudio de impacto ambiental requerido a la luz de las circunstancias específicas de cada caso ${ }^{90}$ y agregó que la obligación de realizar el EIA le corresponde al Estado que realiza la actividad. ${ }^{91}$ Respecto a las obligaciones procedimentales, el fallo reiteró que si el estudio de impacto ambiental confirma que existe un riesgo de un daño transfronterizo significativo el Estado que planea llevar a cabo la tiene la obligación de "notificar y consultar en buena fe a los Estados potencialmente afectados, cuando esto sea necesario para determinar las medidas apropiadas para prevenir o mitigar ese riesgo"92. Dicha obligación forma parte del derecho internacional general y no puede ser excluido mediante un tratado ${ }^{93}$.

\section{Análisis de la contribución de la CIJ}

Podemos sistematizar la jurisprudencia de la $\mathrm{CIJ}$ en tres principales "olas" o tendencias ${ }^{94}$. La primera tendencia está conformada por los casos contenciosos del Canal de Corfú, Ensayos Nucleares I y el obiter dictum de Barcelona Traction. Durante esta etapa la CIJ reconoció ciertos conceptos y principios del Derecho Internacional general que serán aplicados en casos medioambientales en el futuro $0^{95}$. Entre ellos se encuentra la obligación a los Estados de no permitir que sus territorios sean utilizados para ocasionar un perjuicio en el territorio de otro Estado (sic utere tuo ut alienum non laedas) y el reconocimiento de las obligaciones internacionales erga omnes. Asimismo, es importante mencionar que en esta ola se consideraba indispensable la existencia de

Certain Activities carried out by Nicaragua in the border area (Costa Rica v. Nicaragua) \& Construction of a Road in Costa Rica along the San Juan River (Nicaragua v. Costa Rica), I.C.J., Judgment 16 december 2015, pár. 104.

Certain Activities carried out by Nicaragua in the border area (Costa Rica v. Nicaragua) \& Construction of a Road in Costa Rica along the San Juan River (Nicaragua v. Costa Rica), I.C.J., Judgment 16 december 2015, pár. 153

Certain Activities carried out by Nicaragua in the border area (Costa Rica v. Nicaragua) \& Construction of a Road in Costa Rica along the San Juan River (Nicaragua v. Costa Rica), I.C.J., Judgment 16 december 2015, pár. 104.

Certain Activities carried out by Nicaragua in the border area (Costa Rica v. Nicaragua) \& Construction of a Road in Costa Rica along the San Juan River (Nicaragua v. Costa Rica), I.C.J., Judgment 16 december 2015, pár. 108.

De acuerdo a la clasificación de Jorge E. Viñuales. En VIÑUALES, VIÑUALES, Jorge. The Contribution of the International Court of Justice to the Development of International Environmental Law: A Contemporary Assessment, Fordham International Law Journal, Volume 32, Issue 1, Article 14, 2008, p. 235.

Fitzmaurice, Malgosia, The International Court of Justice and International Environmental Law. En: The Development of International Law by the International Court of Justice, editado por Tams, Christian y Sloan, James, Oxford University Press, 2013, p. 373. 
un daño al Estado (componente estatal), puesto que todavía no se reconocía la protección del medio ambiente por su valor intrínseco.

La segunda tendencia está conformada por Gabcikovo-Nagymaros, la opinión consultiva sobre las Armas Nucleares, y la orden en Ensayos Nucleares II. Habían transcurrido varios años desde la primera ola y la preocupación de los Estados por los problemas medioambientales se incrementó, generando así un cambio de percepción ${ }^{96}$. A lo largo de esta tendencia la CIJ, además de reafirmar y consolidar los conceptos desarrollados anteriormente, amplió el campo de la protección ambiental. Durante esta gran ola el medio ambiente como tal adquirió un valor por sí mismo y surgió la obligación de carácter consuetudinario de protegerlo (componente no estatal) ${ }^{97}$.

En la actualidad nos encontramos en la tercera tendencia, conformada por Plantas de Celulosa, y los casos acumulados Ciertas actividades llevadas a cabo por Nicaragua en la zona fronteriza y Construcción de una carretera en Costa Rica a lo largo del río San Juan. La CIJ ha definido ciertas normas jurídicas específicas del DIA, tales como la obligatoriedad de la elaboración del EIA cuando exista el riesgo de ocasionar un daño transfronterizo, y a influenciar en la interpretación de las mismas sobre la base de los principios generales consolidados en la segunda ola ${ }^{98}$.

\section{Conclusión}

En el presente trabajo hemos querido demostrar que la Corte Internacional de Justicia tiene un papel importante en la evolución del Derecho Internacional Ambiental. La contribución de la CIJ es consecuencia de un largo proceso que se sustenta en los conceptos y principios de Derecho Internacional reconocidos en sus primeros fallos. Si bien dichos aportes son "modestos" en comparación con otras áreas del derecho, la Corte contribuyó a que el DIA se convierta en un área independiente del Derecho Internacional en poco tiempo ${ }^{99}$.

Gracias al rol de la CIJ, podemos afirmar que hoy en día existe una obligación consuetudinaria de asegurar que las actividades realizadas dentro de la jurisdicción y

96 Fitzmaurice, Malgosia, The International Court of Justice and International Environmental Law. En: The Development of International Law by the International Court of Justice, editado por Tams, Christian y Sloan, James, Oxford University Press, 2013, p. 362.

Viñuales, Jorge, The Contribution of the International Court of Justice to the Development of International Environmental Law: A Contemporary Assessment, Fordham International Law Journal, Volume 32, Issue 1, Article 14, 2008, p. 246.

Fitzmaurice, Malgosia, The International Court of Justice and International Environmental Law. En: The Development of International Law by the International Court of Justice, editado por Tams, Christian y Sloan, James, Oxford University Press, 2013, p. 370.

Viñuales, Jorge, The Contribution of the International Court of Justice to the Development of International Environmental Law: A Contemporary Assessment, Fordham International Law Journal, Volume 32, Issue 1, Article 14, 2008, p. 258. 
control de los Estados respeten el medio ambiente de otros Estados y el ambiente de las áreas que se encuentran fuera de cualquier control nacional. Esta obligación, de acuerdo con el principio de prevención, aplica de manera general a todas aquellas actividades que puedan ocasionar daños transfronterizos y, para cumplirla, los Estados tienen que ejercer la debida diligencia para prevenir el eventual daño. Este deber de diligencia incluye la elaboración de un EIA antes del inicio de dichas actividades, así como el deber de notificación y consulta de buena fe a los Estados potencialmente afectados. 